\title{
Experimental investigation of five parallel plane jets with variation of Reynolds number and outlet conditions
}

\author{
Tomas Daubner ${ }^{1, *}$, Jens Kizhofer $^{1}$, and Mircea Dinulescu ${ }^{1}$ \\ ${ }^{1}$ Apex Research B.V., Apex Research Laboratory, Westeinde 10 2275AD Voorburg, The Netherlands
}

\begin{abstract}
This article describes an experimental investigation in the near field of five parallel plane jets. The study applies 2D Particle Image Velocimetry (PIV) for ventilated and unventilated jets, where ventilated means exiting into a duct with expansion ratio 3.5 and unventilated means exiting to the free atmosphere. Results are presented for Reynolds numbers 1408, 5857 and 10510. The Reynolds number is calculated for the middle channel and is based on the height of the nozzle (channel) equivalent diameter $2 \mathrm{~h}$. All characteristic regions of the methodology to describe multiple interacting jets are observed by the PIV measurements - converging, merging and combined. Each of the five parallel channels has an aspect ratio of 25 defined as nozzle width (w) to height (h). The channels have a length of 185 times the channel height guaranteeing a fully developed velocity profile at the exit from the channel. Spacing between the single plane jets is 3 times the channel height. The near field of multiple mixing jets is depended on outlet nozzle geometry. Blunt geometry of the nozzle was chosen (sudden contraction).
\end{abstract}

\section{Introduction}

Main focus of the Apex Research Laboratory is the improvement of plate type heat exchangers, the reduction of the pressure drop through the ductwork and the mixing effectiveness in various industrial processes, for example mixing of ammonia in DENOx applications. In the sake of the mixing effectiveness Apex Research Laboratory have developed an experimental setup for understanding the physical phenomena like the mixing of multiple jets. Apex Research Laboratory has published its first paper in the WASET journal in 2017 on the topic "Experimental investigation of plane jets exiting five parallel channels with large aspect ratio" [1], describing the difference between 2 outlet nozzle geometries. Further paper continues in the discussion about multiple jet and its main focus is on the difference in the outlet conditions for multiple jets and its effect on mixing performance.

Multiple parallel plane jets can be found in Heating Ventilating and Air Conditioning systems (HVAC), drying systems and in the combustion chambers. The parameters based on which is necessary to judge the mixing effectiveness are pressure drop and certain mixing when the jets generate a desired velocity distribution.

Single plane jets have been studied extensively since the works of Schlichting [2]. Following studies mainly focussed on characteristics of jet propagation and pointed out the importance and influence of initial boundary conditions [3-4]. L. Moruz [1] studied the mixing effectiveness and Reynolds stress distribution concluding that the outlet geometry is important for this phenomena. We assume that outlet conditions will have also influence on mixing effectiveness express as magnitude - Reynolds stress or as turbulence intensity. This implies that for this paper we have identified following parameters for jet formation: boundary conditions influencing the jet formation are Reynolds number based on channel height (nozzle), nozzle aspect ratio, velocity profiles at exit including mean velocity profile as well as turbulence characteristics and nozzle geometry and spacing between the jets. A Reynolds number dependency will be examined.

Dual parallel jets have been studied by Miller [5] and Nasr [6] beside others. Here, two cases are defined, a ventilated and an unventilated case. The ventilated case has duct downstream from the outlet from the channels, whereas in the unventilated case the jets exit to the free atmosphere. Three main regions have been identified in literature, the converging region, the merging region, and the combined region. When the jets exit the nozzle, a recirculation zone with lower pressure is generated between the jets in the converging region. This low pressure region deflects the jets towards each other. The beginning of the merging region is defined by the merging point, where the recirculation zone vanishes, thus all streamwise velocities are positive. In the merging region the two jets merge together up to the combined point, where only a single combined jet can be identified. For definition of these regions for 2 parallel jets see figure 1 from Nasr [6]. 


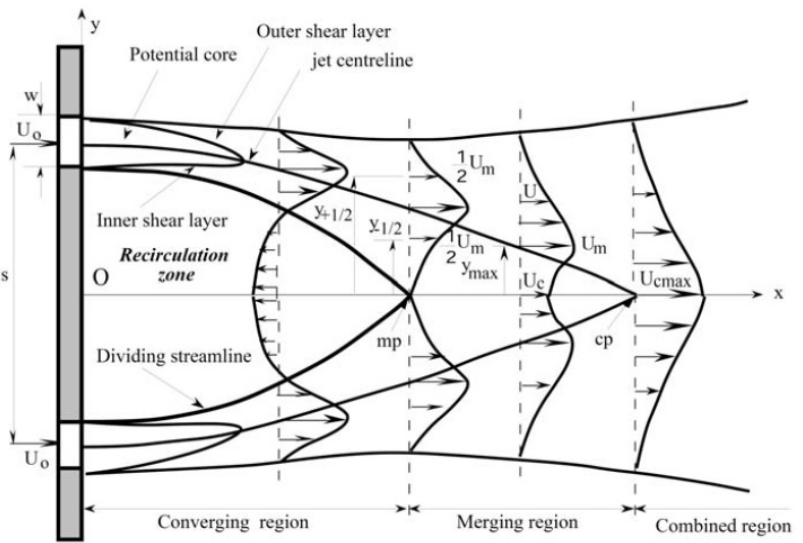

Fig. 1. The definition of the regions and points for two parallel jets [6]

Ghahremanian [7] applied latest measurement equipment like Laser Doppler Anemometry and Particle Image Velocimetry and identified the importance of the consideration of coherent structures in the jet shear layers for the analysis of multiple parallel jets. Furthermore, he discussed the unstable behaviour of the merging points and discussed statistical tools for the prediction of the jet behaviour.

This paper is meant to set up experimental data including the definition of boundary conditions for multiple parallel plane jets exiting to different ambient conditions. For that purpose, chapter 2 describes the experimental set up, the applied measurement techniques like Particle Image Velocimetry and Laser Doppler Anemometry and the measured boundary conditions upstream of the inlet into the five channel geometry as well as the boundary conditions exiting the channels at the nozzle outlet. The third chapter describes and discusses the experimental data with respect to mean velocity field and fluctuating velocity field in terms of Turbulence intensity.

\section{Experimental setup}

Figure 2 shows the outlet from the 5 parallel channels. Figure 3 shows overall view on the facility.

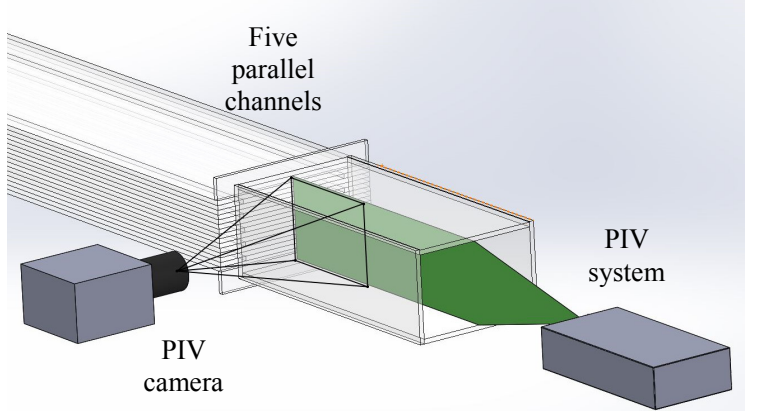

Fig. 2. Closer isometric view for ventilated configuration.

\subsection{Overview of flow facility}

A transparent wind tunnel have been designed to facilitate the air flow distribution in laminar, transient and turbulent regime upstream of the 5 plane parallel channels (see figure 3 ). A centrifugal fan with maximum volume flow rate of $534 \mathrm{~m}^{3} / \mathrm{h}$ and $1.1 \mathrm{~kW}$ electric power drives the air through all the test facility. The volume flow rate is measured with a flow velocity probe from Testo. To control the air flow rate a digital variable power supply is used.

The air is transferred to the measurements position by a round duct, small angle diffuser, square bend, settling chamber, contraction duct, $30^{\circ}$ inclined duct, Apex Delfino Flow Conditioner, five plane parallel channels, nozzle geometries and outlet duct.

The diffuser has a total angle of $12^{\circ}$ for reducing the flow sidewall separation and total pressure drop, the settling chamber has a squared section of $0.3 \mathrm{~m} \mathrm{x} 0.3 \mathrm{~m}$ followed by a contraction duct with an aspect ratio of $1: 3$. The contraction duct is followed by flow straightener of $50 \mathrm{~mm}$ length. The ratio between the honeycomb cell diameter and length guarantees a removal of secondary flow like swirl. The $30^{\circ}$ inclined duct is used to facilitate a non-uniform flow profile just upstream of Apex Delfino Flow Conditioner, which consist of rectangular honeycomb structure and gradually changing fine mesh downstream of the honeycomb, to remove above mentioned influence of $30^{\circ}$ bend.

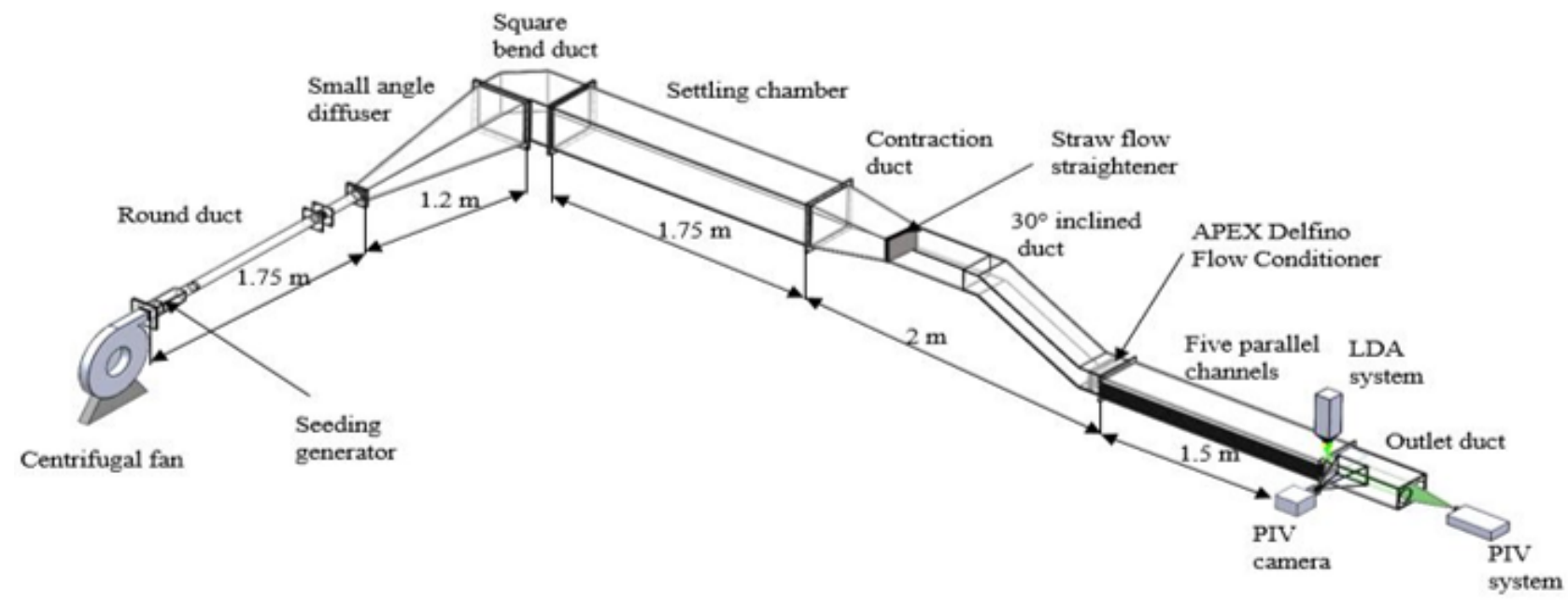

Fig. 3. Transparent flow facility and installed measurement equipment, isometric view 
The five channel geometry consist of transparent plexiglass plates allowing optical measurement techniques. The channel height is $\mathrm{h}=8 \mathrm{~mm}$, width is $\mathrm{w}$ $=200 \mathrm{~mm}$ and the length is $\mathrm{L}=1500 \mathrm{~mm}$ (and hence a large aspect ratio of 25 between channel width / channel height). The non-dimensional spacing ratio between the channels is 3 and the contraction/expansion ratio at inlet/outlet is 3.5 , velocity profile inside the channel is found to be turbulent for lower and higher Reynolds number (see table 1). The Reynolds number is defined as: $R e=(r o \times u \times d \quad h) / \mu$ where $u$ is the mean velocity based on the volume flow rate, $\mathrm{d} h$ is equivalent diameter of the channel which equals with $2 \mathrm{~h}$ and $\mu$ is dynamic viscosity. The outlet conditions are varied between ventilated (figure 2) and unventilated case. To be very clear the unventilated in this report means outlet to the free atmosphere and ventilated means outlet to the ducting as show in figure 2. The duct is 0.5 meter long, the width is equal with width of the channels and the height is shown in figure 4 and is $136 \mathrm{~mm}$.

\subsection{Overview of measurement equipment}

The measurement techniques range from static pressure measurements to laser optical measurement techniques like Particle Image Velocimetry (PIV) or Laser Doppler Anemometry (LDA). For that purpose, a seeding generator introduces DEHS particles in the range of 1 $\mu \mathrm{m}$ into the flow immediately downstream of the fan, see figure 3.

Laser Doppler Anemometry is applied to measure the upstream velocity profile in the center channel. The system is a 2D Flow Explorer from Dantec Dynamics. The LDA system is equipped with a $150 \mathrm{~mm}$ focal length lens generating a measurement volume of $40 \mu \mathrm{m}$ in diameter and $200 \mu \mathrm{m}$ in length. The system is traversed on an ISEL traverse with a positioning accuracy of $\approx 7 \mu \mathrm{m}$. The analysis is performed in the BSA Flow Software. The stop criteria are defined with a measurement length of $30 \mathrm{~s}$ for each point resulting in about 30000 samples or a data rate of 1000 bursts/s

2D Particle Image Velocimetry is applied at the outlet duct to identify the topological behaviour of the five parallel plane jets. The seeding particles are illuminated in the xz-plane at $\mathrm{y}=0.5 \mathrm{w}$ by a $145 \mathrm{~mJ}$ dual cavity laser equipped with a Dantec light sheet optics. The pulse length of the laser is 9 ns to avoid displacement of particles during the illumination. The scattered light of the particles is recorded by a FlowSense EO $4 \mathrm{M}$ double framing camera running at 10 $\mathrm{Hz}$. The separation time between the images is adapted to the jets velocity to yield a maximum displacement of 10 Pix. The number of double frames is 200 resulting in a measurement time of $20 \mathrm{~s}$ per geometry and Reynolds Number. The analysis of the particle images is performed in Dynamic Studio with the "Adaptive PIV" routine. The Cross Correlation algorithm automatically chooses an interrogation area size with respect to number of particles. The minimum number of particles are chosen to be 6/interrogation area. For increased accuracy in shear flows, the algorithm deforms the interrogation area to cover rotation, strain and shear. Erroneous vectors are detected by the N-Sigma outlier detection method with $\mathrm{N}=6$. N-Sigma method is based on computation of mean, RMS and $\sigma$, from series of data and subsequently reject all samples lying more than $\mathrm{N}$ times $\sigma$ from the mean. The scaling is $12 \mathrm{Pix} / \mathrm{mm}$. The resulting vector field has a vector pitch of 16 Pix $(1.3 v e c t o r s / \mathrm{mm})$. Instantaneous picture is shown in figure 4 and figure 5.

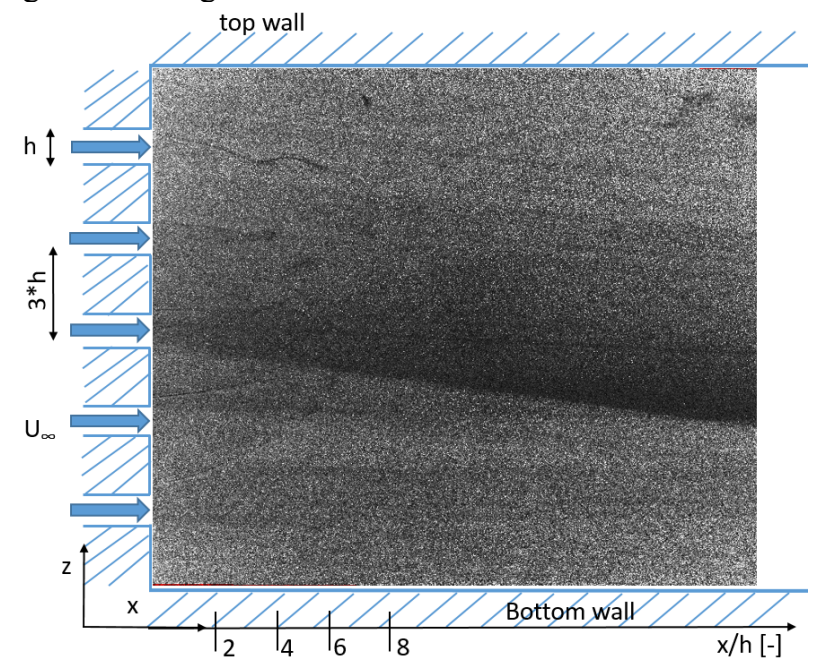

Fig. 4. Example of raw instantaneous PIV image for ventilated configuration for $\mathrm{Re}=1408$

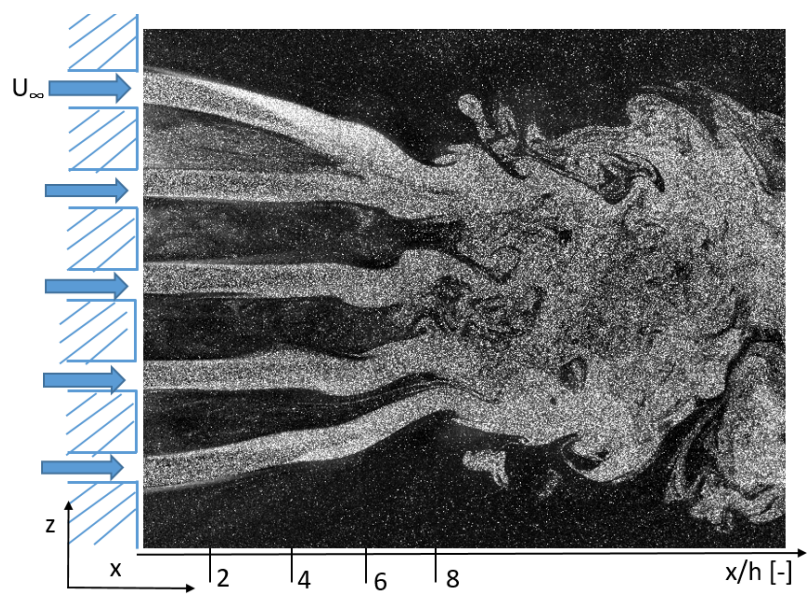

Fig. 5. Example of raw instantaneous PIV image for unventilated configuration for $\mathrm{Re}=1408$

\subsection{Inlet flow conditions}

As stated in literature, the analysis of multiple jets is very sensitive to inlet and boundary flow conditions. Thus, the exact knowledge of those conditions is necessary. The installation of the Apex Delfino Flow Conditioner makes the velocity profile more uniform and reduces the fluctuations to about $5 \%$ at the inlet into the channel model. The streamwise velocity profile at the outlet from the middle channel is shown in figure 6, normalized streamwise velocity profile is shown figure 7 as well as turbulence intensity in figure 8 . Both Figures were acquired by applying LDA measurement technique. Table 1 shows the cases for which the measurement was taken. At $\operatorname{Re}=1408$, the turbulence intensity is very low, indication a laminar flow field. At $\mathrm{Re}=5857$ and 10510 , 
the turbulence intensity in the centre of the channel reach similar amplitudes (5\%).

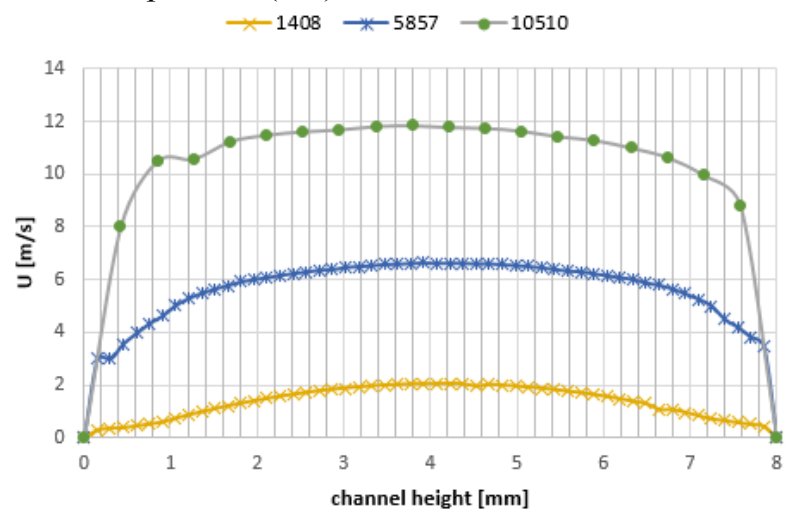

Fig. 6. Streamwise velocity profile for the middle channel for different $\mathrm{Re}$

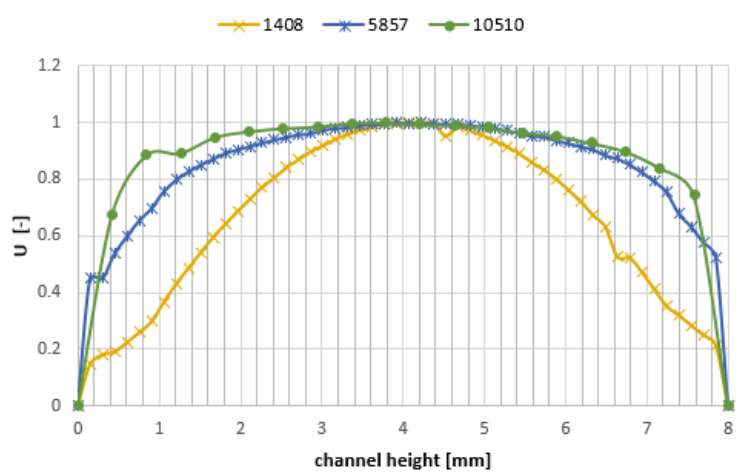

Fig. 7. Normalized streamwise velocity profile for the middle channel for different Re

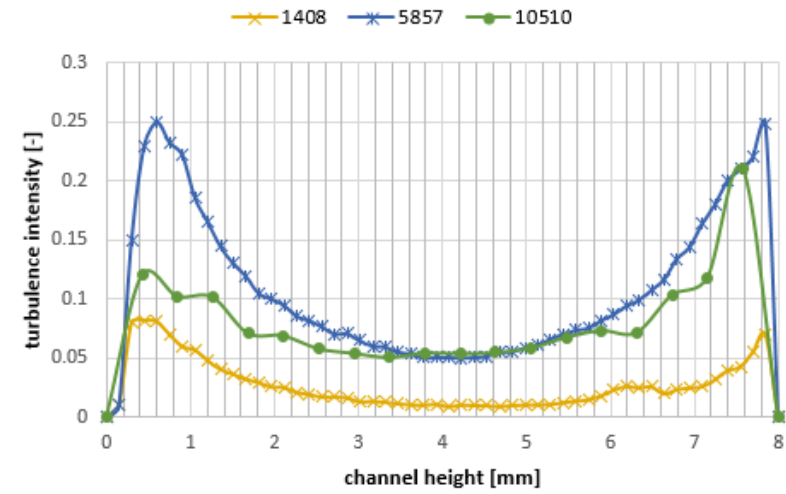

Fig. 8. Turbulence intensity for the middle channel for different Re

Table 1. Measurement characteristics at position 10 times $\mathrm{h}$ upstream from the nozzle outlet

\begin{tabular}{|c|c|c|c|}
\hline $\begin{array}{c}\mathrm{v} \text { mean } \\
{[\mathrm{m} / \mathrm{s}]}\end{array}$ & $\operatorname{Re}$ & $\begin{array}{c}\mathrm{Tu}_{\mathrm{x}} \min \\
{[\%]}\end{array}$ & $\begin{array}{c}\mathrm{Tu}_{\mathrm{x}} \max \\
{[\%]}\end{array}$ \\
\hline 1.32 & 1408 & 1 & 8 \\
\hline 5.49 & 5857 & 5 & 21 \\
\hline 9.85 & 10510 & 5 & 25 \\
\hline
\end{tabular}

\section{Results and discussion}

\subsection{Streamwise velocity}

Figures (9-13) show the normalized positive streamwise mean velocity field (contour plot) and absolute velocity vectors (pitch is 2 times $h$ ) for different Reynolds numbers. Main coordinate system can be found in these figures as well. The scale bar for these figures ranges from $\mathrm{u}=0$ up to $\mathrm{u}=\mathrm{U}_{\infty}$. Where $\mathrm{U}_{\infty}$ is the exiting velocity of the center jet. Scale is for of dimensionless streamwise velocity.
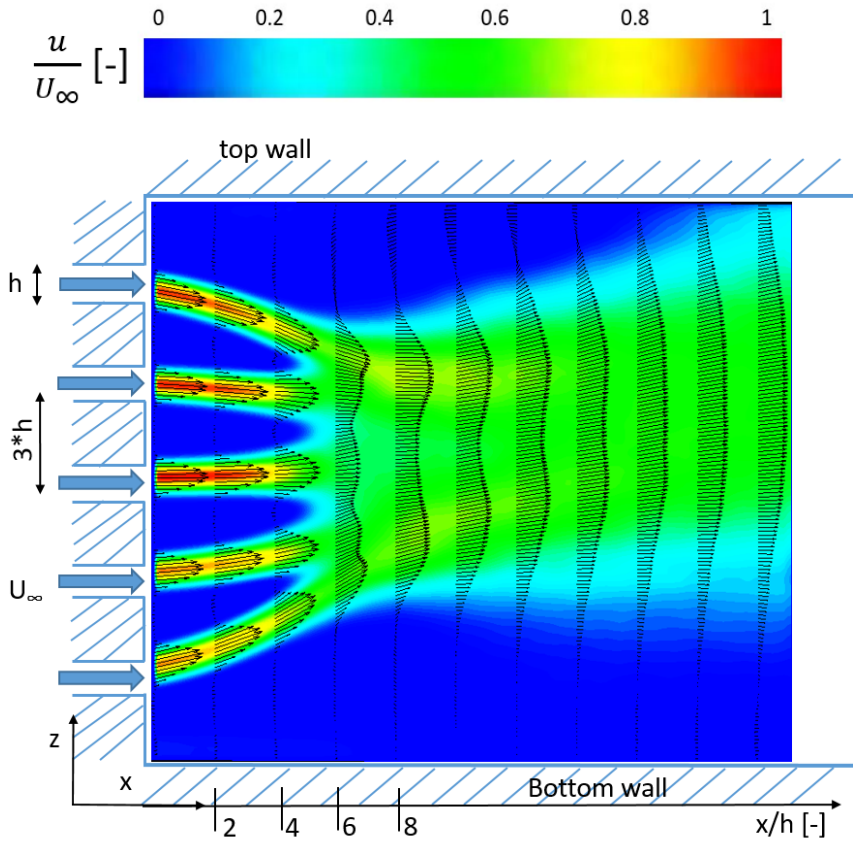

Fig. 9. Ventilated configuration $\mathrm{Re}=1408$

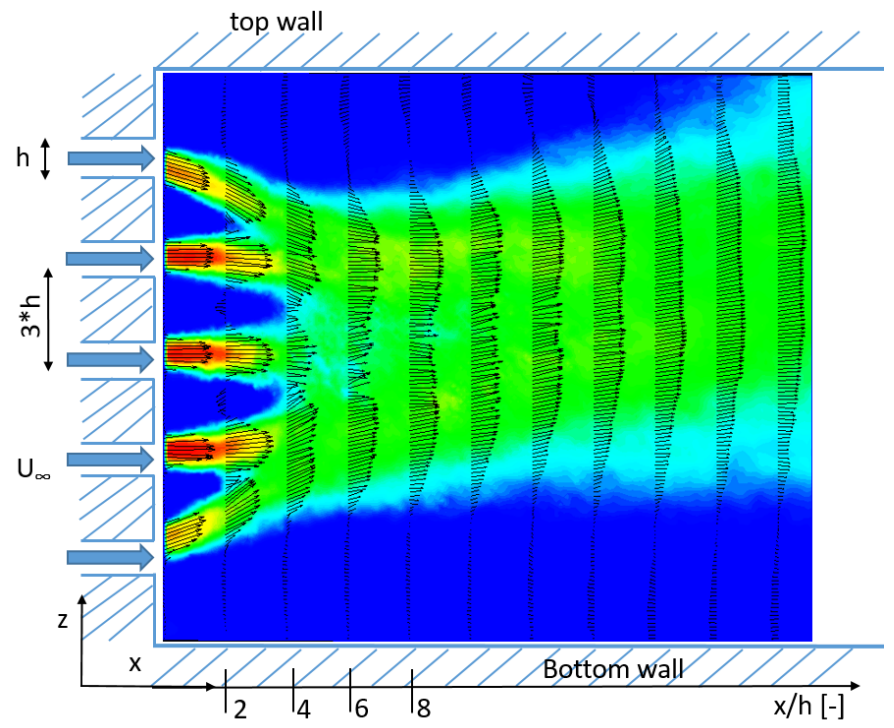

Fig. 10. Ventilated configuration $\mathrm{Re}=5857$ 


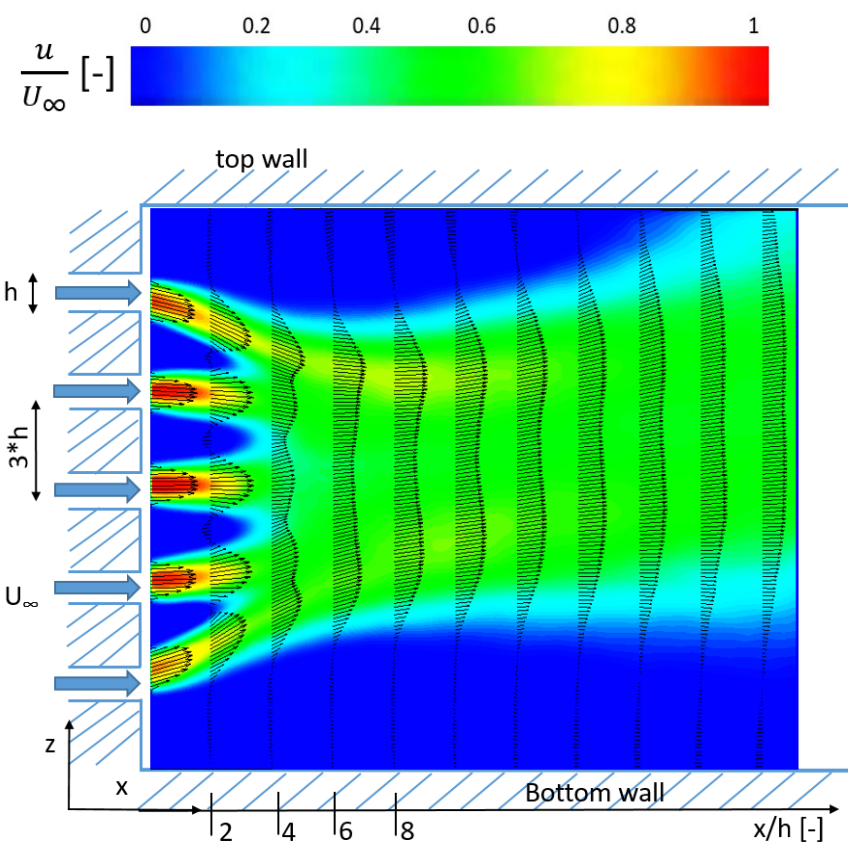

Fig. 11. Ventilated configuration $\mathrm{Re}=10510$

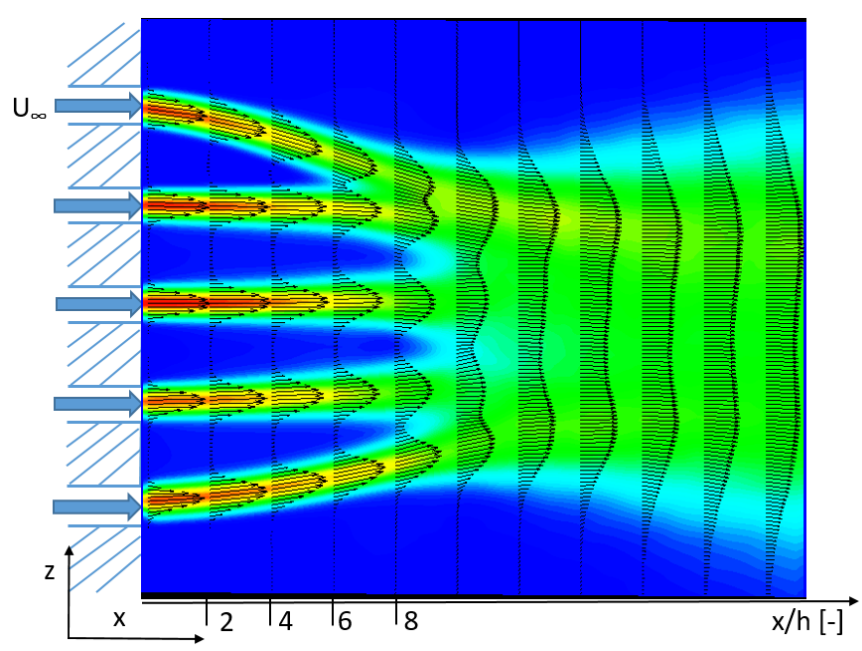

Fig. 12. Unventilated configuration $\mathrm{Re}=1408$

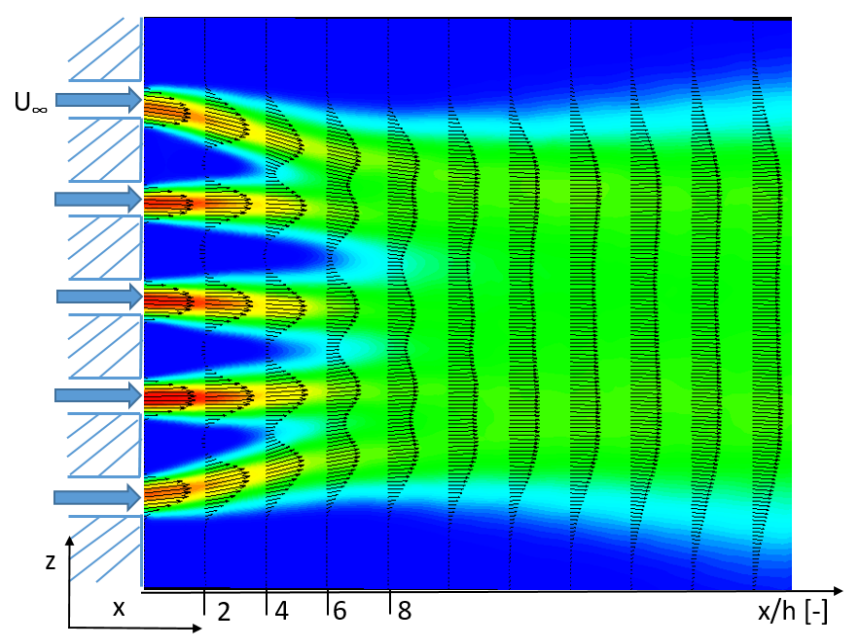

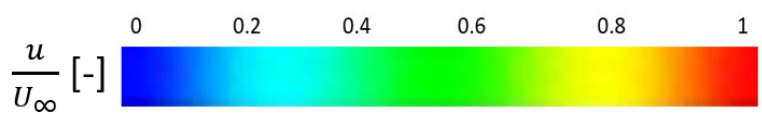

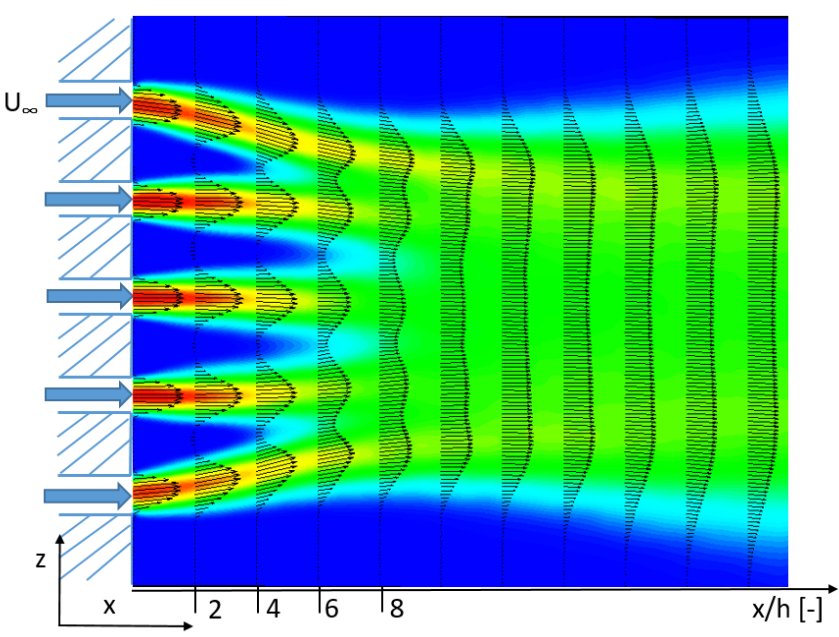

Fig. 14. Unventilated configuration $\mathrm{Re}=10510$

Distance from the nozzle outlet to the merging points also called converging region tends to decrease with increase of the Reynolds number for both configurations. This is valid for laminar and transient flow. After transition to turbulent flow, the flow patterns stabilize and does not change anymore. This distance is decreased for outer jets for both configurations. We have observed that for the ventilated configuration this distance is shorter in comparison with unventilated configuration, where this finding is independent of the Reynolds number.

Big difference is in $\mathrm{x} / \mathrm{h}=4$ for unventilated configuration the multiple jets are more pronounced which is confirming that the converging region is shortened. The mean merging point for ventilated case is at $\mathrm{x} / \mathrm{h}=5$ for $\mathrm{Re}=1408$ and the mean merging point for unventilated case is at $\mathrm{x} / \mathrm{h}=7$ for $\mathrm{Re}=1408$. This can also be observed by looking at the velocity profiles. In other words, the ventilated case tends to have larger contraction angle, defined as inclination angle of the outer jets. This is caused by the pressure gradient downstream from nozzle outlet. Outer jets seem to have longer penetration length.

\subsection{Turbulence intensity}

Figures (16-21) show the turbulence intensity fields in streamwise direction. The scale ranges from 0 up to $5 \%$ for all figures. Moreover, the figures contain the magnitude of velocity in vector form of presentation.

Fig. 13. Unventilated configuration $\mathrm{Re}=5857$ 


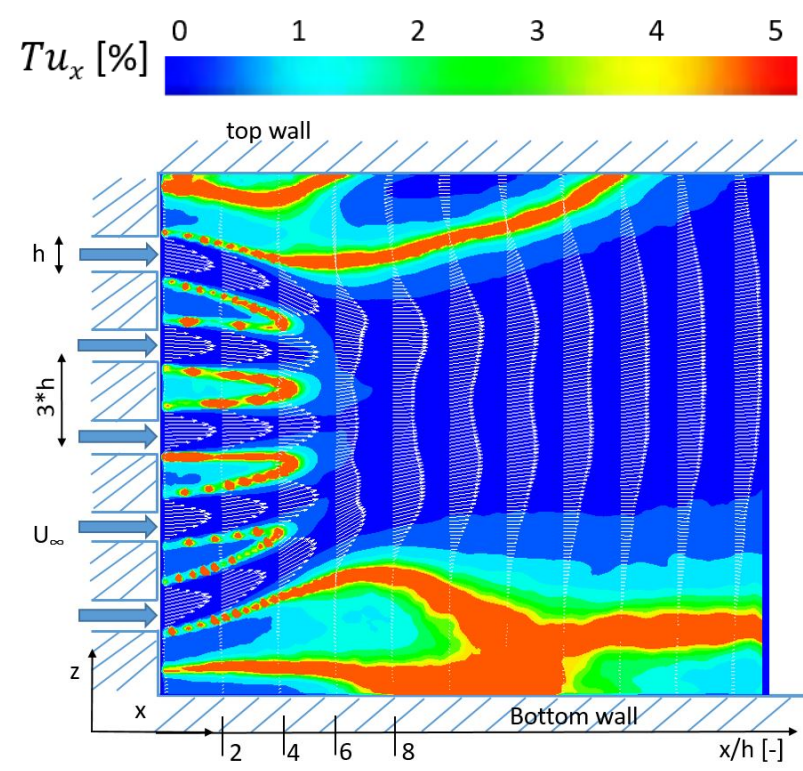

Fig. 15. Turbulence intensity for ventilated case for $\mathrm{Re}=1408$

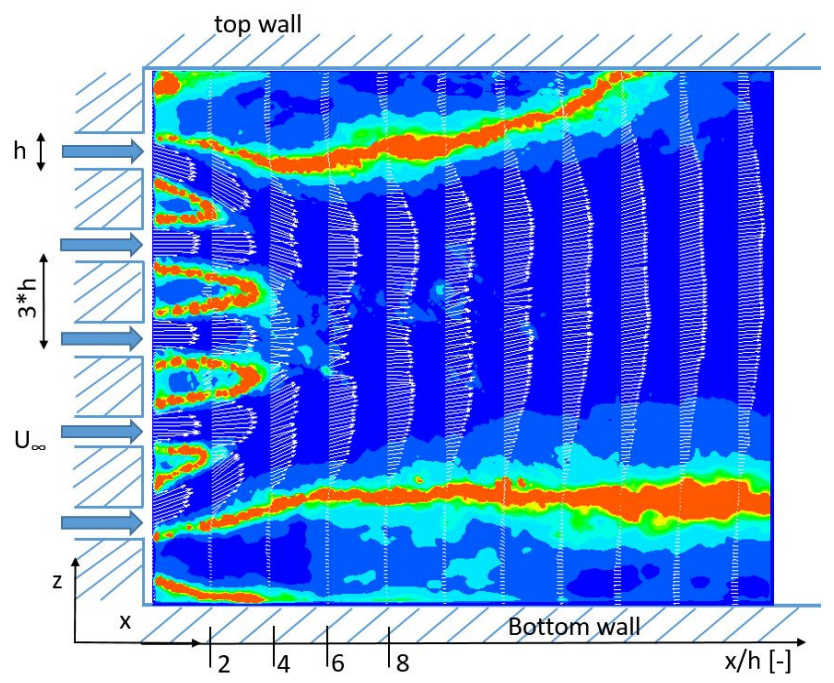

Fig. 16. Turbulence intensity for ventilated case for $\mathrm{Re}=5857$

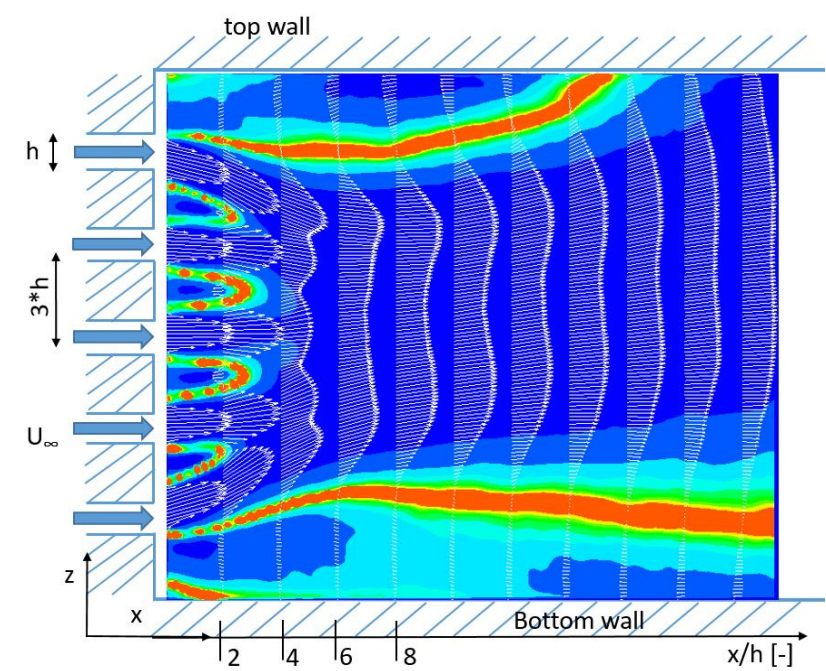

Fig. 17. Turbulence intensity for ventilated case for $\mathrm{Re}=10510$

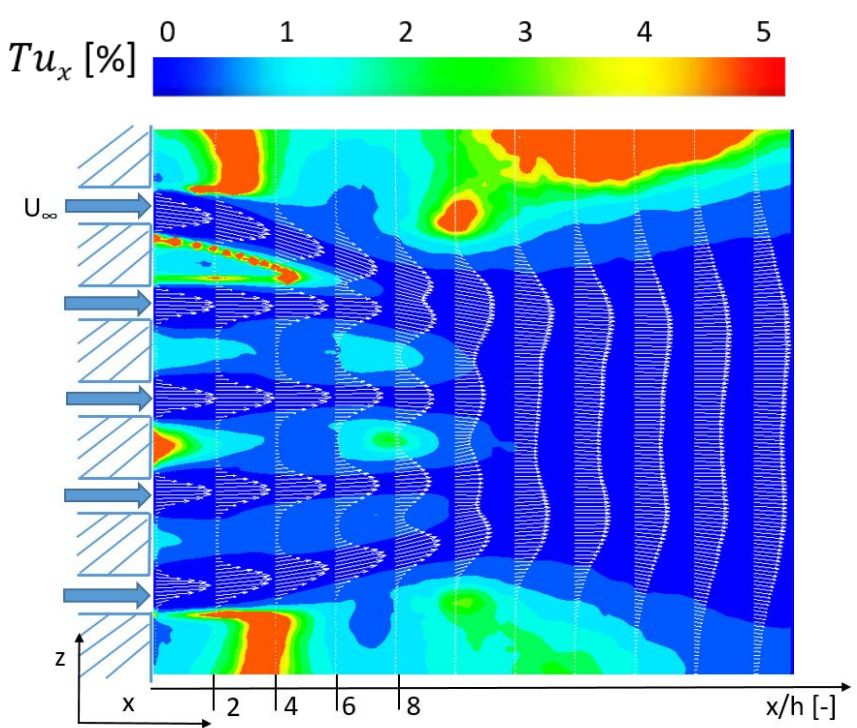

Fig. 18. Turbulence intensity for unventilated case for $\mathrm{Re}=1408$

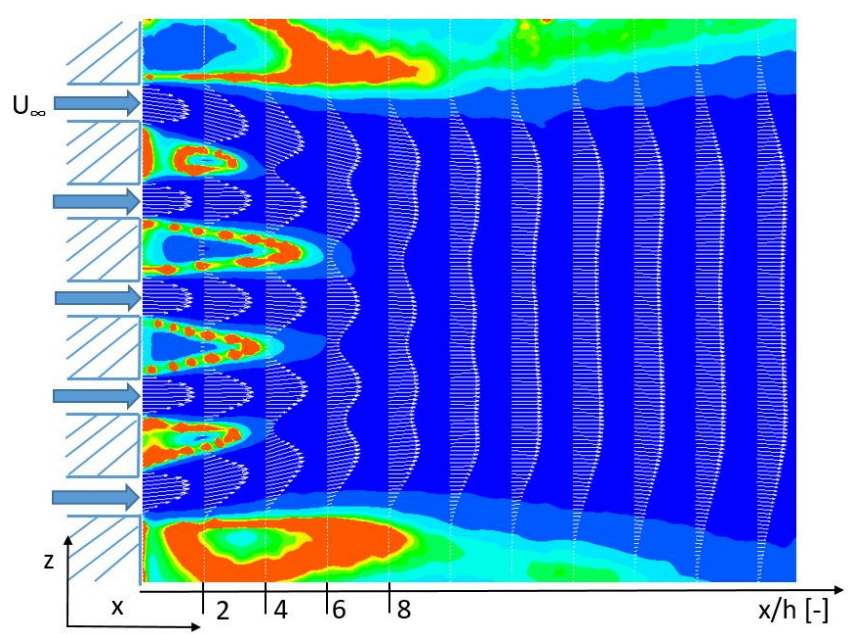

Fig. 19. Turbulence intensity for unventilated case for $\mathrm{Re}=5857$

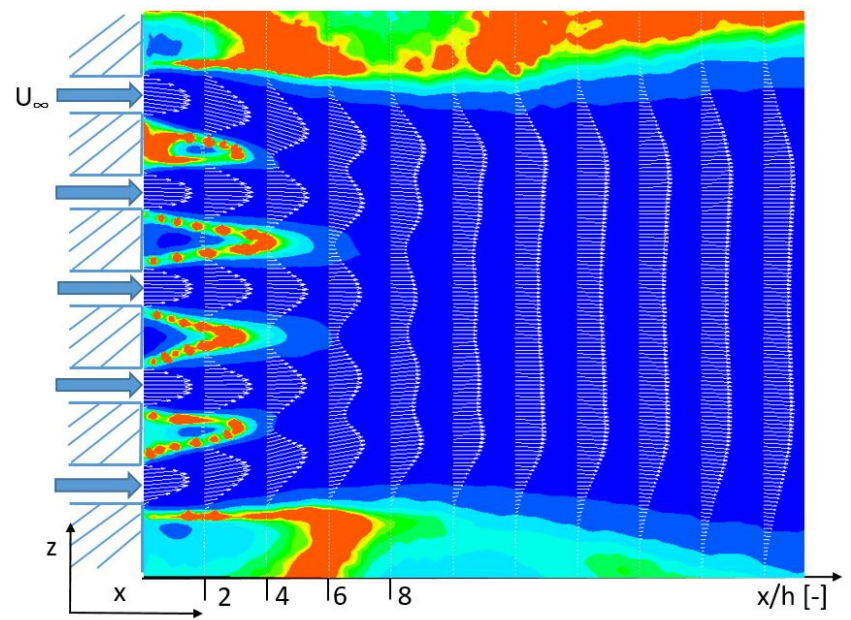

Fig. 20. Turbulence intensity for unventilated case for $\mathrm{Re}=10510$ 
In the ventilated case, recirculation zones on top and bottom wall are generated resulting in a larger tilt angle of the outer jets compared with the unventilated case. These larger angles cause shorter converging regions, which can be observed by the shorter recirculation zones between the jets. Another difference between the configurations clear from the following turbulence intensity figures is that the border between recirculation zone and the jet flow is clearly more visible for ventilated configuration while in ventilated configuration the border is more disperse or missing. In other words, in the unventilated case the jet flow attracts the ambient atmospheric air while in the ventilated configuration the jet flow recirculates the air inside of the duct. The backward phasing step on top and bottom impact the flow differently in un/ventilated case.

\subsection{Turbulence intensity in section and in point}

Figure 21 and figure 22 show $\mathrm{z}$ component of the velocity as a function of time at position $\mathrm{x} / \mathrm{h}=4$ from outlet of the middle jet for $\mathrm{Re}=5857$. The fluctuations are larger for ventilated configuration. Figure 23 shows turbulence intensity at position $\mathrm{x} / \mathrm{h}=4$ from the outlet for $\mathrm{Re}=5857$. The middle jets have larger turbulence intensity than outer jets. For unventilated case the turbulence intensity is more pronounced.

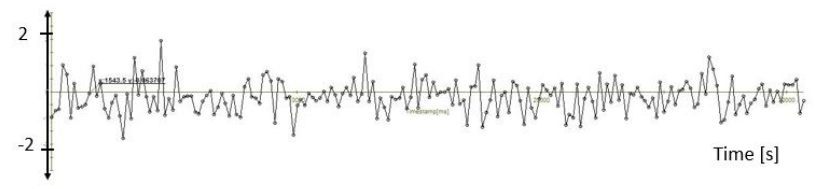

Fig. 21. - Unventilated - Outlet from middle jet at $\mathrm{x} / \mathrm{h}=3$ for $\mathrm{Re}=5857$

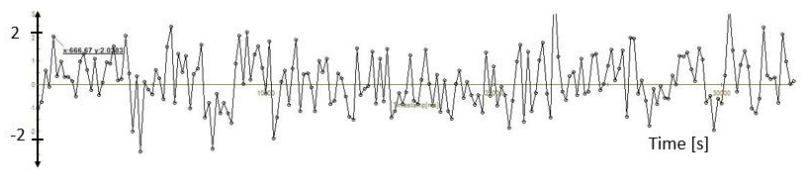

Fig. 22. - Ventilated - Outlet from middle jet at $\mathrm{x} / \mathrm{h}=3$ for $\mathrm{Re}=5857$

\section{Conclusion}

In the ventilated case, recirculation zones on top and bottom wall are generated resulting in a larger tilt angle of the outer jets compared with the unventilated case. This effect is independent of Reynolds number. These larger angles cause shorter converging regions, which can be observed by the shorter recirculation zones between the jets. The mean merging point for ventilated case is at $\mathrm{x} / \mathrm{h}=5$ and the mean merging point for unventilated case is at $\mathrm{x} / \mathrm{h}=7$ for $\mathrm{Re}=1408$. After turbulence flow is reached the merging point stays constant. The merging point is not changing from $\mathrm{Re}=5857$ to $\mathrm{Re}=10510$.

The large turbulence intensity of the ventilated configuration indicates strong mixing in comparison with lower turbulence intensity of the unventilated configuration. Laser Optical Measurement equipment is well suited for the analysis of such complicated velocity fields as multiple interacting jets.

Based on turbulence intensity figures the further research can be done on examining outer shear layer of multiple parallel jets.

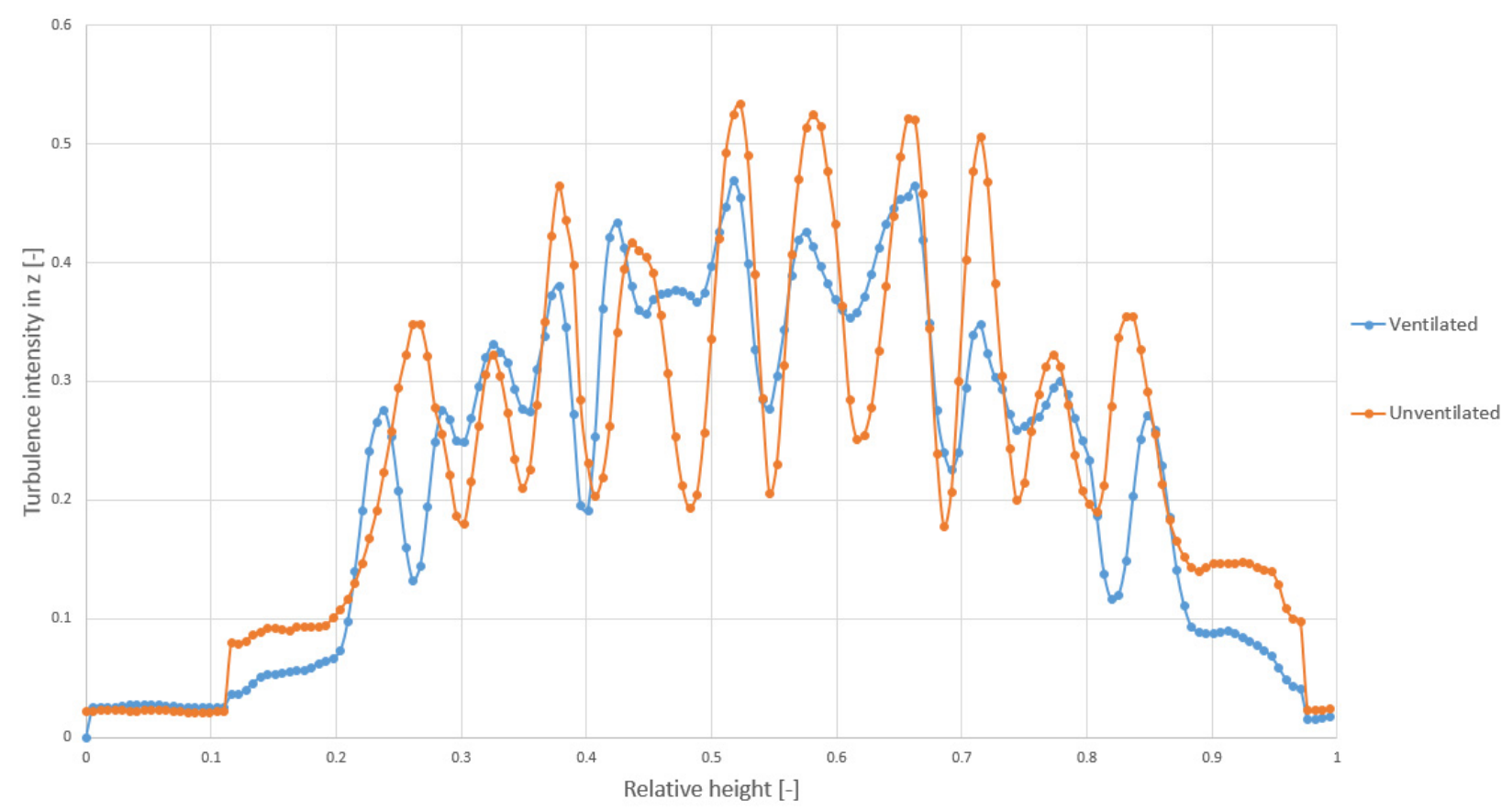

Fig. 23. Turbulence intensity in section for $\mathrm{Re}=5857$ 


\section{References}

1. L. Moruz, J. Kitzhofer, M. Dinulescu, International Journal of Mechanical, Aerospace, Industrial, Mechatronic and Manufacturing Engineering, Experimental investigation of plane jets exiting five parallel channels with large aspect ratio, 11(4), 801 - 808, (2017)

2. H. Schlichting, Laminare Strahlausbreitungs (ZAMM 13, 260, 1933)

3. R.C. Deo, PhD. thesis, Experimental investigations of the influence of Reynolds number and boundary conditions on a plane air jet, University of Adelaide, Australia, (2005)

4. R. C. Deo, Physics of Fluids, The influence of Reynolds number on a plane jet, 20, 75-108, (2008)

5. D.R. Miller, E.W. Comings, Journal of Fluid Mechanics, Force-momentum fields in a dual-jet flow, 7 (2), 237-256, (1960)

6. A. Nasr, J.C.S Lai, International Journal of Transport Phenomena, The effects of nozzle spacing on the development of two parallel plane jets, in International Journal of Transport Phenomena 2 (1), pp. $57-70,2000$

7. S. Ghahremanian, $\mathrm{PhD}$. Thesis, A near-field study of multiple interacting jets: Confluent jets, Linköpimg University, Institute of Technology, Sweden, (2014) 